\title{
Computational Fluid Dynamics Model of Thermal Microenvironments of Corals
}

\author{
$\underline{\text { R. H. Ong }}{ }^{\text {a }}$, A. J. C. King ${ }^{\mathrm{a}}$, B. J. Mullins ${ }^{\mathrm{a}}$, T. F. Cooper ${ }^{\mathrm{b}}$ and M. J. Caley $^{\mathrm{c}}$ \\ ${ }^{a}$ Fluid Dynamics Research Group, Curtin University, Perth, Western Australia \\ ${ }^{\mathrm{b}}$ Australian Institute of Marine Science and UWA Oceans Institute, Perth, Western Australia \\ ${ }^{\mathrm{c}}$ Australian Institute of Marine Science, Townsville, Queensland \\ Email: robert.ong@postgrad.curtin.edu.au
}

\begin{abstract}
We present a model which allows the surface temperature of corals to be predicted, based on environmental factors. The simulation uses specially developed Computational Fluid Dynamics (CFD) models which incorporate the coupled dynamics of water flow in porous media, heat transfer, and spectral irradiance. These models were validated against measurements taken under controlled laboratory conditions, for both massive and branching corals. The results of our CFD simulations agree very well with previously reported experimental results and heat transfer theory. The strong agreement between our modelled results and laboratory observations demonstrates the applicability of CFD, to the study of coral bleaching.
\end{abstract}

Keywords: CFD of thermal microenvironment of corals, OpenFOAM 


\section{INTRODUCTION}

Corals are important habitat-forming species and highly influential on which many other species depend. However, they can bleach in response to stressful thermal conditions which often leads to subsequent mortality. Coral bleaching was first reported back in 1984 and the number of reported cases of major bleaching has grown substantially since. Coral bleaching is caused by a variety of stressors as highlighted by Brown (1997), such as cold water bleaching (Muscatine et al., 1991; Coles and Fadlallah, 1991; Kobluk and Lysenko, 1994), increased or decreased salinity (Van Woesik et al., 1995; Davies et al., 1997; Berkelmans and Oliver, 1999), and sedimentation and turbidity (Jell and Flood, 1978; Hubbard, 1986); but the combination of both elevated sea-surface temperature and solar irradiance is widely acknowledged to be the most important trigger for coral bleaching. Other environmental factors though such as low flow, low salinity and low water turbidity can contribute to the bleaching responses by corals. These parameters vary considerably on coral species and morphologies, which can potentially determine the extent and variation of the fluid flow and mass and heat transfer in corals, and ultimately their susceptibility to bleaching.

Although numerous laboratory and field experiments have now been conducted, the reasons corals bleach are not fully understood; laboratory studies can be confounded by sensitivity of most coral species to laboratory conditions and the difficulties inherent in accurately simulating the complexity and variability of oceanic conditions, while, field-based approaches are typically costly and are difficult to generalise from because of uncontrolled natural variation that may be relevant only to specific sites. Numerical methods on the other hand can be used to solve physics associated with coral bleaching including a wide range of scales of fluid motion, heat transfer, and the morphology of the corals.

Here we present the development of a Computational Fluid Dynamics (CFD) model and solvers for steady state and transient simulations of coral environments in the laminar flow regime. These model and solver were validated using a published case study Jimenez et al. (2008).

\section{PHYSICAL AND MATHEMATICAL MODELS}

The living tissue of corals forms a thin layer over the outermost few millimeters of its calcium carbonate skeleton. These two morphological regions - the tissue layer and the skeletal matrix - are treated separately here in terms of modeling their thermal properties. We explore these properties in both branching and hemispherical coral morphologies (Figure 1). The model configurations and computational domain are shown in Figure 2 (a) and (b).
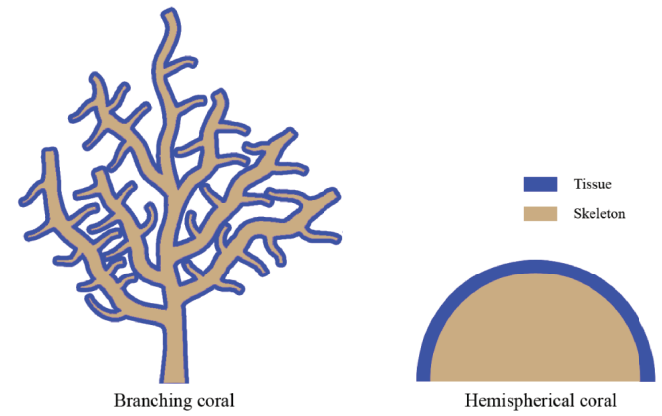

Figure 1. The distribution of living tissue and skeletal matrix in branching and hemispherical corals

Seawater density increases in pressure and temperature in a flow chamber. These increases, however are very small and can be ignored and the flow treated as incompressible. The mass continuity, momentum, and energy governing equations for viscous, incompressible single phase flow through a porous medium 

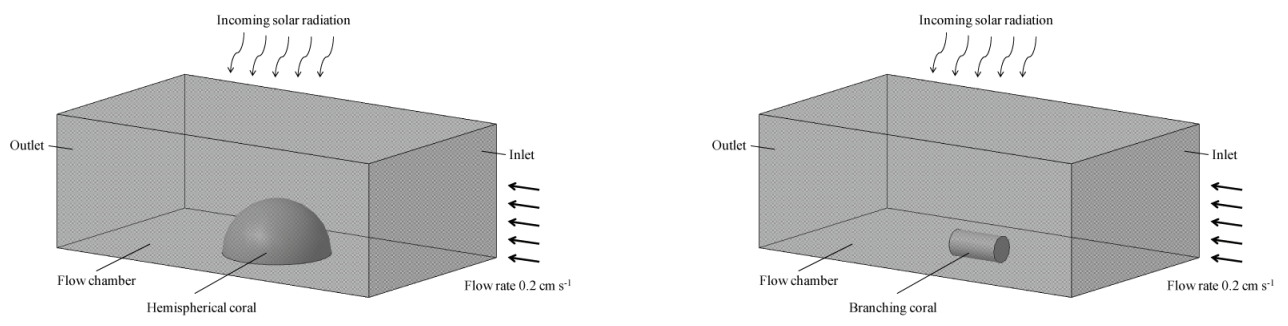

Figure 2. Conceptual representations for both models and experimental set up for (a) hemispherical (b) branching

can be written as

$$
\begin{aligned}
& \nabla \cdot \mathbf{U}=0 \\
& \frac{\partial}{\partial t}(\gamma \mathbf{U})+\mathbf{U} \cdot \nabla \mathbf{U}=\nu \nabla^{2} \mathbf{U}-\frac{\nabla p}{\rho}+S_{\mathrm{i}} \\
& \frac{\partial}{\partial t}(T)+\nabla \cdot(\mathbf{U} T)=\alpha \nabla^{2} T+\frac{\alpha}{k} S_{\mathrm{h}}
\end{aligned}
$$

where $\mathbf{U}$ is the velocity vector, $\gamma$ is porosity, $\nu$ is kinematic viscosity, $p$ is pressure, $k$ is thermal conductivity, $\alpha$ is the thermal diffusivity and $S_{h}$ accounts for heat sources (such as the incident radiation on the tissue, $Q_{\text {rad }}$ ). The flow sink term, $S_{i}$ consists of two parts, a viscous loss term and inertial loss term, which is given by

$$
S_{i}=-\left(\nu D_{\mathrm{ij}}+\frac{1}{2}|\mathbf{U}| F_{\mathrm{ij}}\right) \mathbf{U}
$$

where $D_{\mathrm{ij}}$ and $F_{\mathrm{ij}}$ are represented as the scalars $D$ and $F$, better known as Darcy-Forchheimer coefficients. The Darcy and Forchheimer coefficients which represent viscous energy and kinetic energy losses are respectively given by

$$
\begin{aligned}
& D=\frac{150(1-\gamma)^{2}}{d^{2} \gamma^{3}} \\
& F=\frac{1.75(1-\gamma)}{d \gamma^{3}}
\end{aligned}
$$

To describe the temperature distribution in the porous coral skeleton, we assumed an instantaneous local thermal equilibrium whereby $T_{\mathrm{c}}=T_{\mathrm{f}}=T$ (where $T_{c}$ and $T_{f}$ are the temperatures of the coral and the fluid, respectively). Because tissue occupies an insignificant volume compared to the skeleton (and due to the lack of scientific data available), the porosity of the tissue was assumed to be identical to the skeletal porosity. Hence, averaged material properties for the porous coral skeleton were calculated using

$$
\begin{aligned}
& \rho_{\mathrm{m}}=(1-\gamma) \rho_{\mathrm{c}}+\gamma \rho_{\mathrm{f}} \\
& C_{\mathrm{p}_{\mathrm{m}}}=(1-\gamma) C_{\mathrm{p}_{\mathrm{c}}}+\gamma C_{\mathrm{p}_{\mathrm{f}}} \\
& k_{\mathrm{m}}=(1-\gamma) k_{\mathrm{c}}+\gamma k_{\mathrm{f}}
\end{aligned}
$$

where $C_{p}$ denotes heat capacity. These values were then used to calculate thermal diffusivity, $\alpha$.

$$
\alpha=\frac{k}{\rho C_{p}}
$$


Accounting for a thermal boundary layer, the energy equation can be rewritten in terms of the effective thermal diffusivity, $\alpha_{\text {eff }}$

$$
\frac{\partial}{\partial t}(T)+\nabla \cdot(\mathbf{U} T)=\alpha_{\mathrm{eff}} \nabla^{2} T+\frac{Q_{\mathrm{rad}}}{\rho C_{\mathrm{p}}}
$$

We conducted a simple laboratory experiment to estimate the mean bulk and true densities and the porosity of coral skeletons to provide input data for our models. The skeletal samples were sectioned into defined volumes, dried in an oven at $105^{\circ} \mathrm{C}$, then weighed to determine the skeletal bulk density $\left(\rho_{b}=\frac{m}{V}\right)$. Representative samples of each species used by Jimenez et al. (2008) were accessed (except for the hemispherical coral Cyphastrea seraillia, which was unavailable). Porosity values were then calculated given

$$
\gamma=1-\frac{\rho_{\mathrm{b}}}{\rho_{\mathrm{t}}}
$$

where $\rho_{\mathrm{b}}$ and $\rho_{\mathrm{t}}$ are the bulk and true density of corals, respectively. The estimated (and assumed) porosity values for each of the species examined by Jimenez et al. (2008) are presented in Table 1.

Table 1. Estimated skeletal porosities of four coral species

\begin{tabular}{lc}
\hline \hline Species Name & Skeletal and Tissue Porosity \\
\hline Porites lobata & $0.475 \pm 0.003$ \\
Stylophora pistillata & $0.431 \pm 0.003$ \\
Cyphastrea seraillia & $0.500^{*}$ \\
Seriatopora hystrix & $0.405 \pm 0.003$ \\
\hline
\end{tabular}

* Species not available. Realistic value for massive coral assumed

\section{GRID INDEPENDENCE ANALYSIS}

In our simulations we used hexahedral and polyhedral grids with approximately 900,000 cells depending on the shapes and sizes of the coral. For the purpose of grid independence analysis, the number of grids was varied from 150,000 to $1,000,000$ cells with a constant time step $(\triangle t=1.0)$. As the grid was refined, the truncation error in the solutions should have diminished and consequently the solutions should have approached the asymptotic convergence value.

\section{MODEL VALIDATION}

We used the laboratory experiments of Jimenez et al. (2008) to validate the accuracy of our CFD models. Their study employed two different scenarios, steady-state and transient-state temperature experiments. In the steady-state experiments, a steady inlet flow was maintained at $0.2 \mathrm{~cm} \mathrm{~s}^{-1}$ with the corals exposed to constant solar irradiance levels ranging from around 500 to $950 \mathrm{~W} \mathrm{~m}^{-2}$. The transient experiments in contrast were done to observe the effect of temperature changes under changing solar irradiance levels. Corals were held in darkness for 5 minutes before a dark-light shift was imposed, with an irradiance of $600 \mathrm{~W} \mathrm{~m}^{-2}$. All of their temperature measurements were estimated using thermocouples, or by inserting microsensors connected to a data logger into the coral tissue.

\section{NUMERICAL ASPECTS}

The finite-volume method was employed to solve the governing equations in the computational domain. The run-time discretisation schemes in OpenFOAM were used to discretised using Gaussian integration and flux interpolation, the latter of which may be computed using a variety of centered and normalised variable (NV) scheme (OpenCFD Ltd., 2010). In our model, the schemes implemented for both convection divergence and heat diffusion (Laplacian) terms were the bounded second order linear upwind and the unbounded second order linear limited differencing schemes, respectively. The first time derivative term of the transient time scheme implemented in our model used the Euler scheme, which is the 
first order, bounded and implicit scheme available in OpenFOAM for solving ordinary differential equations (OpenCFD Ltd., 2010). The generalised geometric-algebraic multi-grid (GAMG), smoother GaussSeidel, and preconditioned bi-conjugate gradient (PBiCG) were used to discretised pressure, velocity, and temperature governing equations, respectively. The semi-implicit method for pressure-linked equation (SIMPLE) algorithm and merged PISO-SIMPLE (PIMPLE) algorithm were used for steady-state and transient simulations, respectively. The SIMPLE algorithm calculates pressure on a staggered grid from velocity components by applying an iterative procedure coupled with the Navier-Stokes equations (Ferziger and Peric, 1999), meanwhile, the PIMPLE algorithm combines the SIMPLE algorithm then uses pressure implicit with splitting the operators (PISO) algorithm to rectify the second pressure correction and correct both velocities and pressure explicitly (Ferziger and Peric, 1999; Versteeg and Malalasekera, 1995).

The prescribed initial and boundary conditions were nescessary for simulating the flow conditions that correspond to Jimenez et al. (2008). The setting of the initial and boundary conditions for velocity, pressure, and temperature are established as shown in Table 2.

Table 2. Initial and boundary conditions for models

\begin{tabular}{ccccccc}
\hline \hline variable & initial & wall & top & floor & inlet & outlet \\
\hline pressure $(\mathrm{p})$ & $0 \mathrm{~Pa}$ & $\mathrm{~S}$ & $\mathrm{~S}$ & $\mathrm{ZG}$ & $\mathrm{ZG}$ & $\mathrm{FV}$ \\
velocity $(\mathrm{U})$ & $0.002 \mathrm{~m} \mathrm{~s}^{-1}$ & $\mathrm{~S}$ & $\mathrm{~S}$ & $\mathrm{FV}$ & $\mathrm{FV}$ & $\mathrm{ZNG}$ \\
temperature $(\mathrm{T})$ & $26^{\circ} \mathrm{C}$ & $\mathrm{S}$ & $\mathrm{S}$ & $\mathrm{FV}$ & $\mathrm{FV}$ & $\mathrm{ZG}$ \\
\hline
\end{tabular}

S: Slip, ZG: Zero Gradient, ZNG: Zero Normal Gradient, FV: Fixed Value

\section{RESULTS}

\subsection{GRID INDEPENDENCE ANALYSIS}

In most cases, the number of cells needed to reach steady-state coral tissue warming was approximately 600,000 to 800,000 cells (Figure 3 ). These result suggest that the grids used in our models were sufficiently fine to capture these heating dynamics, hence, further refinement of the grid would be unlikely to change the results presented here.

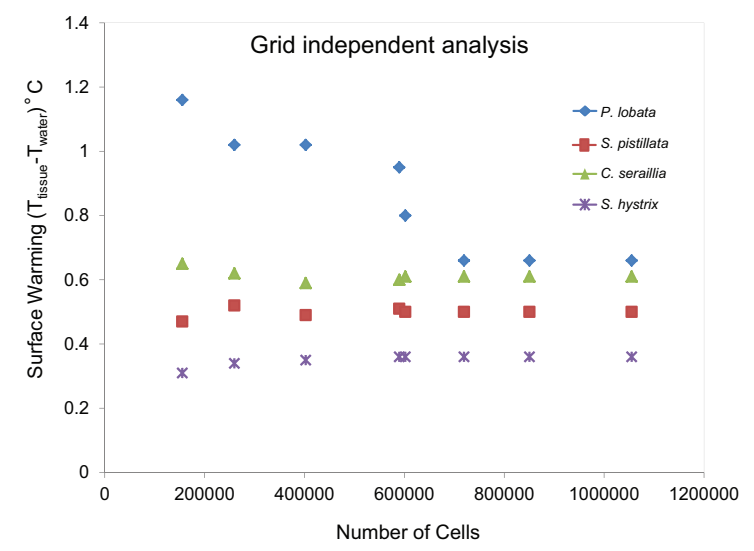

Figure 3. Grid independence analysis based on varying number of cells

\subsection{STEADY STATE SIMULATIONS}

Both the experimental data of Jimenez et al. and the CFD models agreed well with a linear relationship between coral surface warming and irradiance; consistent with heat transfer theory. Surface warming 
rates for the hemispherical coral P.lobata linearly increased with increasing irradiance, whereas surface warming rates of the branching coral $S$. pistillata did not increase with increasing irradiance. This difference between the two species was due to the temperature rise being highly dependent on the coral's surface area-to-volume ratio. The correlation of between irradiance and surface warming of our modeled results was $\mathrm{R}^{2}=0.99$ for both hemispherical and branching corals, as opposed to the experimental results which yield $\mathrm{R}^{2}=0.59$ and 0.92 for branching and hemispherical corals, respectively (Figure 4 ).
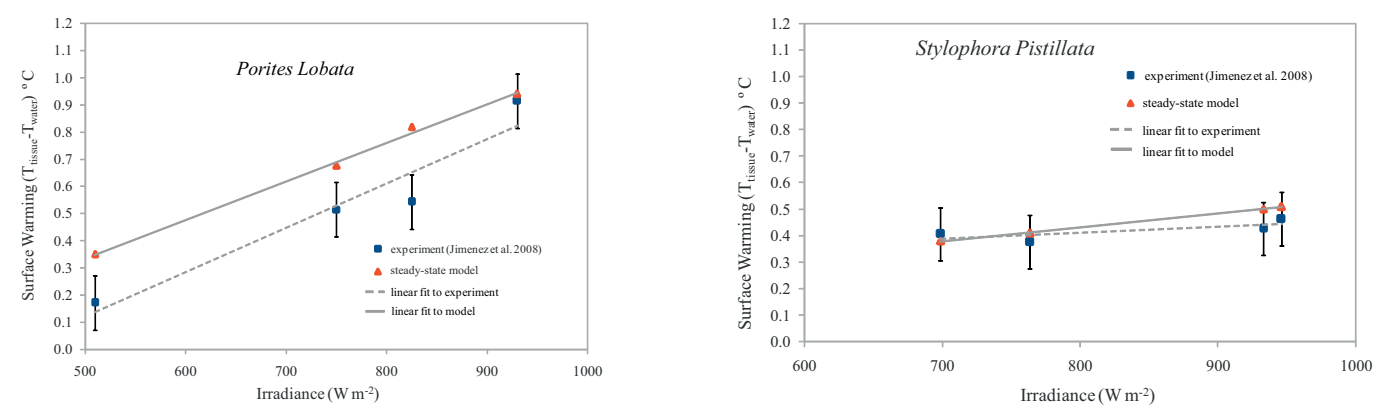

Figure 4. Comparisons of simulated and experimental warming of hemispherical and branching corals under direct solar irradiance

\subsection{TRANSIENT SIMULATIONS}

The time taken for hemispherical and branching corals to reach thermal equilibrium, after the dark-light shift imposed, was 225 and $115 \mathrm{~s}$, respectively. This in equilibration time constant arose from differences in the surface-area-to-volume ratio between hemispherical and branching corals (Figure 5). The smaller temperature increase experienced by the branching coral compared to the hemispherical coral is consistent with theoretical expectations and experimental results.
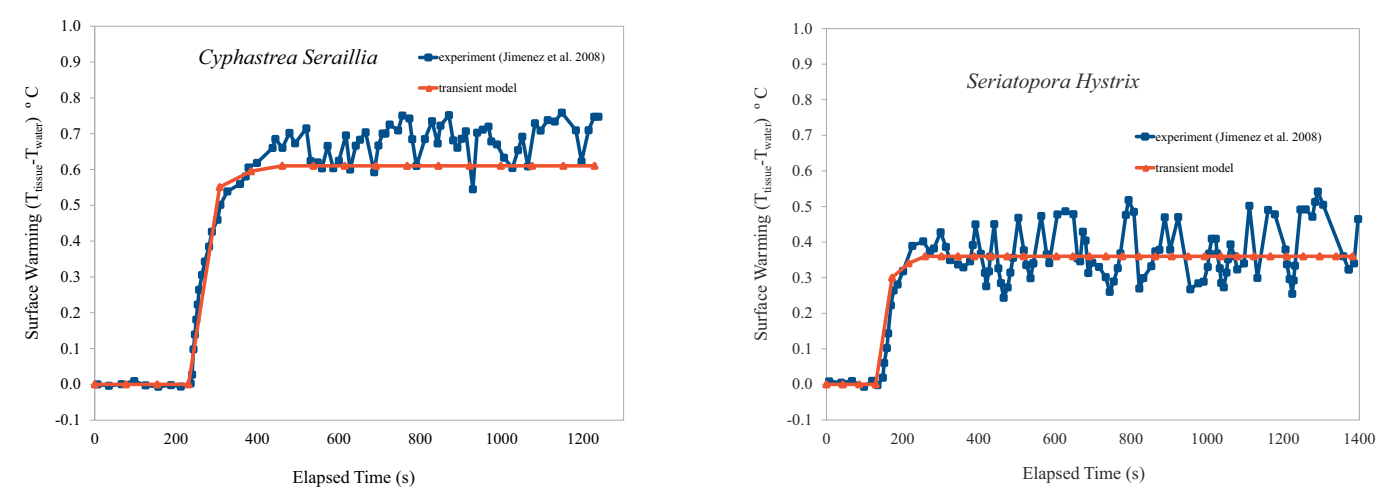

Figure 5. Surface warming in response to a dark-light shift of hemispherical and branching corals

\subsection{VELOCITY PATTERNS}

The suspension flow in hemispherical coral at $\mathrm{Z}=0[\mathrm{~m}]$ is clearly shown by the contours and velocity magnitudes which conforms with theoretical expectations (Figure 6), however, more experiments and validations are needed to establish the physical properties of coral (i.e. porosity and permeability) which may play a significant role in determining coral surface temperatures.

\subsection{PARALLEL PERFORMANCE}

Our computations were performed on a Intel ${ }^{\complement}$ Core $^{\mathrm{TM}}$ i7-860 HT workstation and run in parallel by employing domain decomposition on four cores and eight threads. The fundamental issue when using the 


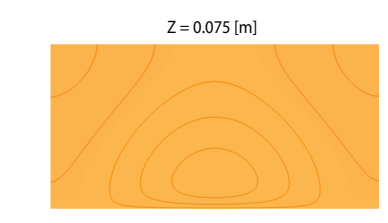

$\mathrm{Z}=0.025[\mathrm{~m}]$

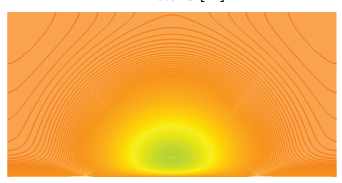

$Z=-0.025[\mathrm{~m}]$

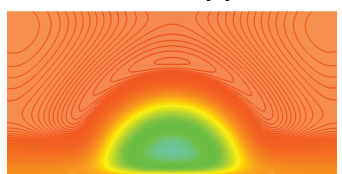

Velocity $(\mathrm{m} / \mathrm{s})$
$\mathrm{Z}=0.05[\mathrm{~m}]$

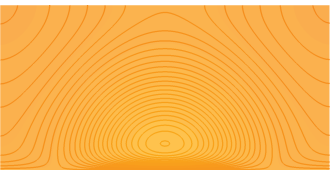

$\mathrm{Z}=0[\mathrm{~m}]$

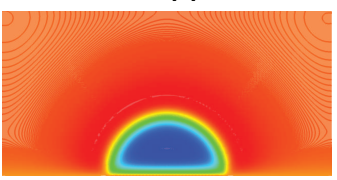

$Z=-0.05[\mathrm{~m}]$

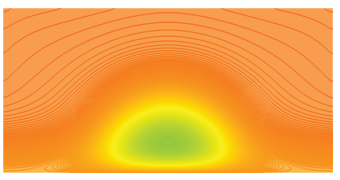

01650.00228

Figure 6. Field of axial velocity at different cross sectional planes perpendicular to flow

multi-processors computer is speedup. Speedup is defined as the ratio of the execution time on a single processor to that on multiple number of processors $N$.

$$
S(n)=\frac{\text { Execution time using one processor }}{\text { Execution time using N processors }}=\frac{t_{s}}{t_{n}}
$$

Message Passing Interface (MPI) was implemented as the communication protocol among the processors. The wall clock times for full simulations of steady-state and transient solvers are presented in Table 3 and 4 , respectively.

Table 3. Speedup performance based on wall clock time for steady-state solver

\begin{tabular}{lccc}
\hline \hline Processor & Number of Cells in each Processors & Wall Time (s) & Speedup \\
\hline 1 & 225,355 & 101,800 & 1.0 \\
2 & 225,417 & 55,586 & 1.83 \\
3 & 225,417 & 34,508 & 2.95 \\
4 & 225,355 & 25,386 & 4.01 \\
\hline
\end{tabular}

Table 4. Speedup performance based on wall clock time for transient solver

\begin{tabular}{lccc}
\hline \hline Processor & Number of Cells in each Processors & Wall Time (s) & Speedup \\
\hline 1 & 218,266 & 103,191 & 1.0 \\
2 & 218,320 & 53,191 & 1.94 \\
3 & 218,320 & 34,627 & 2.98 \\
4 & 218,266 & 25,797 & 4.0 \\
\hline
\end{tabular}

Table 3 and 4 demonstrated that the computational time decreased as more processors were used. A linear speedup was observed when going from one to two CPUs, however, the speedup was significantly reduced afterwards. This was due to the increased in the total amount of processor idle time. Hence, we decided 
not to measure the speedup performance solely based on the CPU time since other factors are critical as well, such as the mesh computation part which cost considerable time on I/O consuming.

\section{CONCLUSION}

In summary, whilst there are still many improvements that still can be made to the current models and solvers, we have demonstrated that CFD models can be a powerful tool for investigating the relationship between the thermal microenvironment of corals and its relationship to coral bleaching. Using CFD techniques enabled us to map many of the parameters of interest (i.e., flow, pressure, temperature, etc) within the interiors of corals that would have been inaccessible by other means. These parameters are related to coral morphology, and are likely prime determinants of mass and heat transfer in corals, and ultimately their sensitivity to bleaching.

\section{ACKNOWLEDGMENT}

The authors acknowledge both iVEC and the Society for Underwater Technology (SUT) for funding. Finally, we acknowledge the OpenFOAM community and developers.

\section{REFERENCES}

Berkelmans, R. and J. Oliver (1999). Large-scale bleaching of corals on the Great Barrier Reef. Coral Reefs 18(1), 55-60.

Brown, B. (1997). Coral bleaching: causes and consequences. Coral Reefs 16, 129-138.

Coles, S. and Y. Fadlallah (1991). Reef coral survival and mortality at low temperatures in the Arabian Gulf: new species-specific lower temperature limits. Coral Reefs 9(4), 231-237.

Davies, J., R. Dunne, and B. Brown (1997). Coral bleaching and elevated sea-water temperature in Milne Bay Province, Papua New Guinea, 1996. Marine and Freshwater Research 48(6), 513-516.

Ferziger, J. and M. Peric (1999). Computational Methods for Fluid Dynamics (2nd edition ed.). Springer.

Hubbard, D. (1986). Sedimentation as a control of reef development: St. Croix, USVI. Coral Reefs 5(3), 117-125.

Jell, J. and P. Flood (1978). Guide to the Geology of Reefs of the Capricorn and Bunker Groups, Great Barrier Reef Province with special reference to the Heron Reef. Papers, Department of Geology, University of Queensland 8(3), 1-85.

Jimenez, I., M. Kuhl, A. Larkum, and P. Ralph (2008). Heat budget and thermal microenvironment of shallow-water corals: Do massive corals get warmer than branching corals? Limnology and Oceanography 53(4), 1548-1561.

Kobluk, D. and M. Lysenko (1994). Ring bleaching in Southern Caribbean Agaricia agaricites during rapid water cooling. Bulletin of Marine Science 54(1), 142-150.

Muscatine, L., D. Grossman, and J. Doino (1991). Release of symbiotic algae by tropical sea anemones and corals after cold shock. Marine ecology progress series. Oldendorf 77(2), 233-243.

OpenCFD Ltd. (2010, August). OpenFOAM - User Guide. OpenCFD Ltd. Version 1.7, http://www.openfoam.com/.

Van Woesik, R., L. De Vantier, and J. Glazebrook (1995). Effects of Cyclone'Joy'on nearshore coral communities of the Great Barrier Reef. MEPS 128, 261-270.

Versteeg, H. and W. Malalasekera (1995). An introduction to computational fluid dynamics: the finite volume method. Longman Scientific \& Technical. 